\title{
Synthesis and in vitro antiproliferative activity of new adamantylthiazolyl-1,3,4-oxadiazoles
}

\author{
Maryam Zahid, ${ }^{1}$ Khawaja A. Yasin, ${ }^{1}$ Tashfeen Akhtar, ${ }^{1}$ Nasim H. Rama, ${ }^{1}$ Shahid Hameed, ${ }^{1, *}$ \\ Najim A. Al-Masoudi, ${ }^{2,}{ }^{*}$ Roberta Loddo, ${ }^{3}$ and Paolo La Colla ${ }^{3}$ \\ ${ }^{1}$ Department of chemistry Quaid-i-Azam University Islamabad-45320, Pakistan \\ ${ }^{2}$ Chemistry Department, College of Basrah, Basrah, Iraq (previous address: FB Chemie, \\ University of Konstanz, P.O. Box 5560, D-78457 Konstanz, Germany) \\ ${ }^{3}$ Department of Biomedical Sciences and Technologies, University of Cagliari, Cittadella \\ Universitaria, 09042 Monserrato (CA), Italy \\ E-mail:shameed@qau.edu.pk; najim.al-masoudi@gmx.de
}

\begin{abstract}
A new series of adamantanyl-1,3-thiazole and 1,3,4-oxadiazole derivatives (6a-1), bearing various aryl groups has been synthesized from adamantan-1-nitrile in four steps. All the compounds were evaluated, in vitro, for antiproliferative activity against a large panel of human tumor-derived cell lines. Compounds 6e exhibited activity against human splenic B-lymphoblastoid (WIL-2NS) and human acute B-lymphoblastic leukemia (CCRF-SB) cell lines with $C_{50}=68$ and $42 \mu \mathrm{M}$, respectively. Compound $\mathbf{6 l}$ showed activity against CCRF-SB cell lines with $C C_{50}=51 \mu \mathrm{M}$. All the other compounds were found inactive.
\end{abstract}

Key words: Adamantan-1-nitrile, antitumor activity, anti-HIV activity, thiazole, oxadiazoles.

\section{Introduction}

Amantadine hydrochloride 1 (1-adamantanamine hydrochloride, Symmetrel $\left.{ }^{\circledR}\right)$ was the first adamantane derivative introduced in medicine as effective therapy ${ }^{1-3}$ against Asian A influenza virus. Among various substituents a growing interest in adamantyl derivatives is gaining prominence because of well known drugs like Rimantadine, Memantine, Adapalene, Adatanserin and others in clinical trials. ${ }^{4,5}$ The pronounced central nervous stimulant and cardiovascular effects of amantadine ${ }^{6}$ necessitated the search for newer more potent and less toxic agents for the control of pandemic influenza viruses. $N$-1-adamantyl-4-aminophthalimide 2 was endowed with anti-HIV-1 and -HIV-2 activities in CEM cell cultures. ${ }^{6}$ Potent anti-HIV-1 activity was recently observed for a series of ( \pm -2-(1-adamantyl-3-alkyl/aryl)thiazolidin-4-ones where these compounds behaved as 


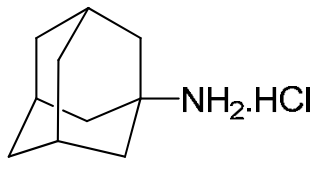

1

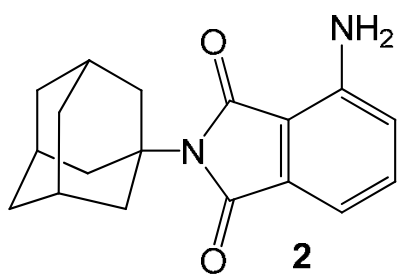

typical non-nucleoside reverse transcriptase inhibitors. ${ }^{7,8}$ Burstein et al. ${ }^{9}$ developed adamantane derivatives, in which the adamantane moiety is chemically linked to a water soluble polyanionic matrix. These derivatives proved to be good inhibitors of replication in early stages of HIV-1. In addition, the activity of some adamantane derivatives has recently improved their use in clinical therapeutic efficacy of interferon/ribavirin combination against hepatitis $C{ }^{10}$ Some other adamantyl derivatives have been used as anti-inflammatory, ${ }^{11-14}$ antimicrobial, $^{15-17}$ antimalarial ${ }^{18}$ and antidepressant $^{19}$ agents as well as inhibitors of $11 \beta$-hydroxysteroid dehydrogenase type 1 (11ßHSD1). ${ }^{20}$

We have recently reported the synthesis and biological activities of various azoles. ${ }^{21-23}$ In the present study, we selected three pharmacophores i.e. 1,3-thiazole, 1,3,4-oxadiazole and adamantyl precursors, to build up potent molecules possessing these three backbones, aiming to investigate their anticancer and antiviral activities.

\section{Results and Discussion}

Adamantan-1-nitrile was selected as starting material for the synthesis of target compounds. The nitrile was converted into thioamide $3(52 \%)$, using $\mathrm{P}_{4} \mathrm{~S}_{10}$ followed by its treatment with ethyl bromopyruvate to afford 4 (80\%). Hydrazinolysis of 4 gave the carbohydrazide-1,3-thiazole 5 in $75 \%$ yield. Heating 5 with substituted benzoic acids in the presence of polyphosphoric acid (PPA) furnished 1,3,4-oxadiazole derivatives 6a-l in 61-66\% yield. The synthetic reactions are summarized in scheme 1. 


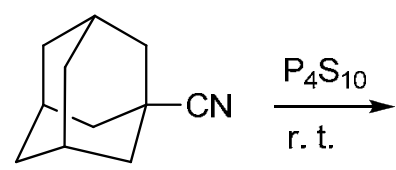

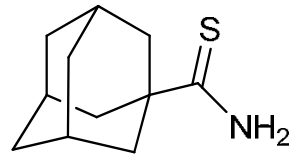

3

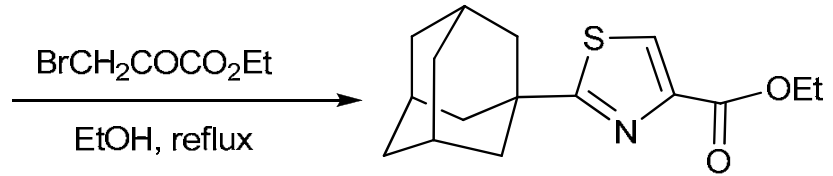

4

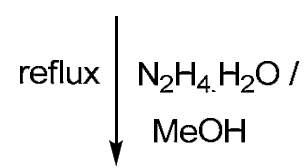

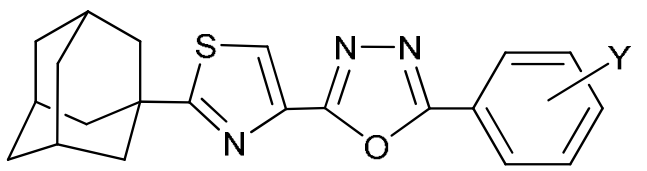

6

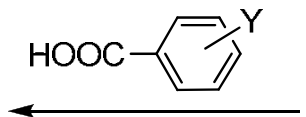

PPA, reflux

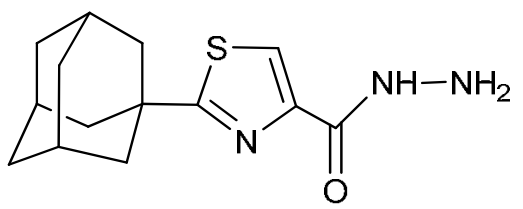

5

a: $Y=4-\mathrm{CH}_{3} ; \mathbf{b}: \mathrm{Y}=3-\mathrm{CH}_{3} ; \quad$ c: $\mathrm{Y}=2-\mathrm{CH}_{3}$;

d: $Y=4-\mathrm{Cl} ; \quad$ e: $Y=3-\mathrm{Cl} ; \quad$ f: $Y=2-\mathrm{Cl}$;

g: $Y=4-B r ; \quad h: Y=3-B r ; \quad i: Y=2-B r ;$

$\mathbf{j}: Y=4-F ; \quad k: Y=3-F ; \quad l: Y=2-F$

Scheme 1. Synthesis of 2-(2-adamantyl-1,3-thiazol-4-yl)-5-aryl-1,3,4-oxadiazoles.

The synthesis of 3 was confirmed in the IR and NMR spectra. In the IR spectrum, the typical sharp absorptions at $v_{\max } 3424$ and $3323 \mathrm{~cm}^{-1}$ characteristic of the primary $\mathrm{NH}_{2}$ group were observed. The ${ }^{1} \mathrm{H}-\mathrm{NMR}$ spectrum exhibited two singlets at $\delta 7.95$ and 7.10 attributed to the $\mathrm{NH}_{2}$ protons. In the ${ }^{13} \mathrm{C}-\mathrm{NMR}$ spectrum, the downfield signal at $\delta 218.9$ was assigned to the thiocarbonyl carbon. Additional support for the formation of $\mathbf{3}$ was obtained by appearance of the molecular ion peak in the mass spectrum at $\mathrm{m} / z$ 195. The structures of compounds 4 and $\mathbf{5}$ were also established using IR and NMR spectroscopy and the molecular mass confirmed by MS. The IR spectra of 4 and 5 exhibited absorptions corresponding to the carbonyl groups at $v_{\max } 1732$ and $1663 \mathrm{~cm}^{-1}$, respectively. In the ${ }^{13} \mathrm{C}$-NMR spectra, the signals at $\delta 161.7$ and 162.2 were attributed to the carbonyl carbon atoms in compound 4 and 5, respectively. In the ${ }^{1} \mathrm{H}-\mathrm{NMR}$ of compound 5, a broad singlet observed at $\delta 8.48$ was assigned to the $\mathrm{NH}_{2}$ group. Additional support for formation of 4 and 5 were obtained by appearence of the molecular ion peaks in the mass spectra at $\mathrm{m} / \mathrm{z} 291$ and 277 , respectively.

The structures of 6a-l were confirmed by the IR, NMR and mass spectra. The IR spectra were characterized by the C-O absorptions in the range $v_{\max } 1262-1102 \mathrm{~cm}^{-1}$, an indicative for the $1,3,4-$ oxadiazole ring formation. In the ${ }^{1} \mathrm{H}$ NMR spectra, four aromatic protons were appeared in the range of $\delta 7.33-8.33 \mathrm{ppm}$. The singlets in the range $\delta 7.88-8.12$ were assigned to $\mathrm{H}-5$ of the thiazole moiety. In the ${ }^{13} \mathrm{C}$-NMR spectra, the resonances in the region $\delta \sim 161.0$ and $\delta \sim 163.0$ were assigned to $\mathrm{C}-2$ and $\mathrm{C}-5$ of the oxadiazole ring, respectively. The carbons of the adamantane moiety were located at the region $\delta$ 28.5-43.1 ppm. Compound 6d was selected for further NMR study. From the gradient ${ }^{24}$ selected HMBC spectrum of $\mathbf{6 d}, \mathrm{H}-5$ of the thiazole ring at $\delta_{\mathrm{H}} 8.09$ showed a ${ }^{3} J_{\mathrm{C}, \mathrm{H}}$ 
couplings with C-2 of the thiazole ring at $\delta_{\mathrm{C}} 183.8$ and C-2 of the oxadiazole ring at $160.9 \mathrm{ppm}$.

Furthermore, a ${ }^{2} J_{\mathrm{C}, \mathrm{H}}$ coupling of the same proton with C-4 of the thiazole ring at $\delta_{\mathrm{C}} 139.2 \mathrm{ppm}$ was also observed.

\section{In vitro antiproliferative activity}

Compounds 6a-l were tested, in vitro, against a large panel of human cell lines derived from hematological $\left[\mathrm{CD}^{+}\right.$human T-cells containing an integrated HTLV-1 genome (MT-4); CD4 ${ }^{+}$ human acute T-lymphoblastic leukemia (CCRF-CEM); human splenic B-lymphoblastoid cells (WIL-2NS); human acute B-lymphoblastic leukemia (CCRF-SB) and solid skin melanoma (SK-28); breast adenocarcinoma (MCF-7); lung squamous carcinoma (SK-MES-1); hepatocellular carcinoma (HepG-2); prostate carcinoma (DU-145)] or normal tissues [lung fibroblasts (MRC-5)]. For comparative purposes, we evaluated the cytotoxic activities of the compounds relative to Doxorubicin.

All compounds were inactive except 6e which showed activity against human splenic Blymphoblastoid cells (WIL-2NS) and human acute B-lymphoblastic leukemia (CCRF-SB) cell lines with $C C_{50}=68$ and $42 \mu \mathrm{M}$, respectively. Compound $\mathbf{6 l}$ exhibited activity against CCRF-SB cell lines with $C C_{50}=51 \mu \mathrm{M}$.

\section{Experimental Section}

General Procedures. Melting points are uncorrected and were measured on a Gallenkamp melting point apparatus (MP-D). The elemental analysis was performed on Leco CHNS-932 Elemental Analyzer, Leco Corporation (USA). N MR spectra were recorded on a Bruker Avance $300 \mathrm{MHz}$ spectrometer with TMS as an internal standard and on the $75 \mathrm{MHz}\left({ }^{13} \mathrm{C}\right.$ ) (scale in $\delta$ ). The multiplicities are expressed as $\mathrm{s}=$ singlet, $\mathrm{bs}=$ broad singlet, $\mathrm{d}=$ doublet, $\mathrm{dd}=$ doublet of doublets, $\mathrm{dt}=$ doublet of triplets, $\mathrm{t}=$ triplet and $\mathrm{m}=$ multiplet. Mass spectra were recorded on Agilent technologies $6890 \mathrm{~N}$ gas chromatograph and an inert mass selective detector 5973 mass spectrometer. The $\mathrm{R}_{f}$-values were determined employing pre-coated silica gel aluminium plates, Kieslgel $60 \mathrm{~F}_{254}$ from Merck (Germany), using $n$-hexane: ethyl acetate (7:3) as an eluent unless otherwise mentioned. Column chromatography was carried out using silica gel 60 (0.063-0.200 $\mathrm{mm}$ ) purchased form Merck. The IR spectra were recorded on FTS $3000 \mathrm{MX}$, Bio-Rad Merlin (Excalibur Model) spectrophotometer.

2-Adamantanethioamide (3). $\mathrm{P}_{4} \mathrm{~S}_{10}(5.33 \mathrm{~g}, 12.00 \mathrm{mmol})$ was stirred at room temperature in EtOH $(25 \mathrm{~mL})$ for $2 \mathrm{~h}$. Adamantane-1-nitrile $(1.0 \mathrm{~g}, 6.20 \mathrm{mmol})$ was added to the above solution and the reaction mixture heated under reflux for $12 \mathrm{~h}$. After completion of the reaction, the solution was concentrated in vacuo, diluted with water and extracted with $\mathrm{CH}_{2} \mathrm{Cl}_{2}(3 \times 50 \mathrm{~mL})$. The combined organic extracts were dried $\left(\mathrm{Na}_{2} \mathrm{SO}_{4}\right)$, filtered and concentrated in vacuo and the yellow liquid was refrigerated. The resulting white crystals were filtered, dried and recrystallized from aq. EtOH to 
give $3(0.63 \mathrm{~g}, 52 \%)$; mp 159-161 ${ }^{\circ} \mathrm{C}$; $\mathrm{R}_{\mathrm{f}}: 0.54$. IR $\left(v_{\max }, \mathrm{KBr}, \mathrm{cm}^{-1}\right): 3424,3323,2907,2848,1656$, 1449, 1384, 1181. ${ }^{1} \mathrm{H}-\mathrm{NMR}\left(\mathrm{CDCl}_{3}\right): \delta 1.79$ (m, 6H, $\left.\mathrm{CH}_{2}-4, \mathrm{CH}_{2}-6, \mathrm{CH}_{2}-10\right), 2.18\left(\mathrm{~m}, 9 \mathrm{H}, \mathrm{CH}_{2}-2\right.$, $\left.\mathrm{CH}-3, \mathrm{CH}-5, \mathrm{CH}-7, \mathrm{CH}_{2}-8, \mathrm{CH}_{2}-9\right), 7.10$ (bs, 1H, N-H), $7.95(1 \mathrm{H}, \mathrm{bs}, \mathrm{N}-\mathrm{H}) .{ }^{13} \mathrm{C}-\mathrm{NMR}\left(\mathrm{CDCl}_{3}\right): \delta$ 28.4, 36.2, 41.7, 45.6 ( $\left.\mathrm{C}_{\text {adamant. }}\right)$; $218.9(\mathrm{C}=\mathrm{S})$. EI-MS (m/z, \%): $195\left(\mathrm{M}^{+}, 80\right), 135$ (100), 121 (5), 107 (15), 93 (27), 60 (16). Anal. calcd. for $\mathrm{C}_{11} \mathrm{H}_{17} \mathrm{NS}$ : C, 67.64; H, 8.77; N, 7.17. Found: C, 67.54; H, 8.72; N, 7.38.

Ethyl 2-adamantyl-1,3-thiazole-4-carboxylate (4). A mixture of 2-adamantanethioamide (3) (0.29 $\mathrm{g}, 1.5 \mathrm{mmol})$ and ethyl bromopyruvate $(0.29 \mathrm{~g}, 1.5 \mathrm{mmol})$ in EtOH $(25 \mathrm{~mL})$ were heated under reflux for $8 \mathrm{~h}$. After cooling, the reaction mixture was concentrated in vacuo, diluted with water and extracted with $\mathrm{CH}_{2} \mathrm{Cl}_{2}(3 \times 25 \mathrm{~mL})$. The combined organic layers were dried $\left(\mathrm{Na}_{2} \mathrm{SO}_{4}\right)$, filtered and concentrated in vacuo to afford 3 as a yellow oil $(0.35 \mathrm{~g}, 80 \%), \mathrm{R}_{\mathrm{f}}: 0.73$. IR $\left(v_{\max }\right.$, film, $\left.\mathrm{cm}^{-1}\right)$ : $3117,2906,2850,1732,1605,1497,1477,1451,1368,1093 .{ }^{1} \mathrm{H}-\mathrm{NMR}\left(\mathrm{CDCl}_{3}\right): \delta 1.40(\mathrm{t}, 3 \mathrm{H}, J=$ $\left.7.2 \mathrm{~Hz}, \mathrm{OCH}_{2} \mathrm{CH}_{3}\right), 1.79\left(\mathrm{~m}, 6 \mathrm{H}, \mathrm{CH}_{2}-4, \mathrm{CH}_{2}-6, \mathrm{CH}_{2}-10\right), 2.18$ (m, 9H, CH${ }_{2}-2, \mathrm{CH}-3, \mathrm{CH}-5, \mathrm{CH}-7$, $\left.\mathrm{CH}_{2}-8, \mathrm{CH}_{2}-9\right), 4.41\left(2 \mathrm{H}, \mathrm{q}, J=7.2 \mathrm{~Hz}, \mathrm{OCH}_{2} \mathrm{CH}_{3}\right), 8.05(1 \mathrm{H}, \mathrm{s}, \mathrm{H}-12) .{ }^{13} \mathrm{C}-\mathrm{NMR}\left(\mathrm{CDCl}_{3}\right): \delta 14.4$ $\left(\mathrm{OCH}_{2} \mathrm{CH}_{3}\right) ; 28.5,36.4,39.8,41.7\left(\mathrm{C}_{\text {adamant. }}\right) ; 61.2\left(\mathrm{OCH}_{2} \mathrm{CH}_{3}\right) ; 125.9\left(\mathrm{C}^{5}{ }_{\text {thiazole }}\right) ; 146.6\left(\mathrm{C}^{4}{ }_{\text {thiazole }}\right)$; $161.7(\mathrm{C}=\mathrm{O}) ; 182.3\left(\mathrm{C}_{\text {thiazole }}^{2}\right)$. EI-MS (m/z, \%): $291\left(\mathrm{M}^{+*}, 90\right), 246$ (100), 135 (45), 121 (3), 107 (9), 93 (15), 71, (50), 45 (10).

2-Adamantyl-1,3-thiazole-4-carbohydrazide (5). Hydrazine hydrate 80\% (5.2 mmol) was added slowly to a stirred solution of ethyl 2-adamantyl-1,3-thiazole-4-carboxylate (4) (0.38 g, $1.3 \mathrm{mmol})$ in $\mathrm{MeOH}(5 \mathrm{~mL})$ and the reaction mixture heated under reflux for $4 \mathrm{~h}$. After cooling, the mixture was concentrated in vacuo, followed by addition of cold water. The precipitated solid was filtered, dried $\left(\mathrm{Na}_{2} \mathrm{SO}_{4}\right)$ and recrystallized from aq. EtOH to give (5) $(0.27 \mathrm{~g}, 75 \%) ; \mathrm{mp} \mathrm{179-181}{ }^{\circ} \mathrm{C} ; \mathrm{R}_{\mathrm{f}} 0.73$ (petroleum ether : acetone; 2:3). IR ( $\left.v_{\max }, \mathrm{KBr}, \mathrm{cm}^{-1}\right): 3424,3323,3184,1663,1541,1491 .{ }^{1} \mathrm{H}-$ NMR $\left(\mathrm{CDCl}_{3}\right): \delta 1.79\left(\mathrm{~m}, 6 \mathrm{H}, \mathrm{CH}_{2}-4, \mathrm{CH}_{2}-6, \mathrm{CH}_{2}-10\right), 2.04\left(\mathrm{~m}, 9 \mathrm{H}, \mathrm{CH}_{2}-2, \mathrm{CH}-3, \mathrm{CH}-5, \mathrm{CH}-7\right.$, $\left.\mathrm{CH}_{2}-8, \mathrm{CH}_{2}-9\right), 4.08$ (bs, 2H, NH$)_{2}, 7.99$ (s, 1H, H-12), 8.48 (bs, 1H, N-H). ${ }^{13} \mathrm{C}-\mathrm{NMR}\left(\mathrm{CDCl}_{3}\right): \delta$ 28.4, 36.4, 39.6, 43.1 ( $\left.\mathrm{C}_{\text {adamant. }}\right) ; 121.8\left(\mathrm{C}_{\text {thiazole }}^{5}\right) ; 147.9\left(\mathrm{C}_{\text {thiazole }}^{4}\right) ; 162.2(\mathrm{C}=\mathrm{O}) ; 182.1\left(\mathrm{C}_{\text {thiazole }}^{2}\right)$. EI-MS (m/z, \%): 277 (M+ं, 95), 246 (100), 219 (10), 179 (5), 135 (47), 121 (3), 107 (9), 93 (15). Anal. calcd. for $\mathrm{C}_{14} \mathrm{H}_{19} \mathrm{~N}_{3} \mathrm{SO}$ : C, 60.64; H, 6.88; N, 15.11. Found: C, 60.35; H, 6.66; N, 15.03 .

\section{General procedure for the synthesis of 2-(2-adamantyl-1,3-thiazol-4-yl)-5-aryl-1,3,4- oxadiazoles (6a-l)}

A mixture of $5(0.50 \mathrm{~g}, 1.8 \mathrm{mmol})$ and substituted benzoic acid (1.8 mmol) was heated at 100-120 ${ }^{\circ} \mathrm{C}$ in presence of excess polyphosphoric acid (PPA) for $4 \mathrm{~h}$. After cooling, the mixture was poured into crushed ice, and neutralized with $5 \%$ aq. $\mathrm{NaHCO}_{3}$ solution. The precipitated solid was filtered and purified using column chromatography (petroleum ether : ethyl acetate; $9: 1$ ).

2-(2-Adamantyl-1,3-thiazol-4-yl)-5-(4-methylphenyl)-1,3,4-oxadiazole $\quad$ (6a). From 4methylbenzoic acid (0.25 g). Yield: $0.45 \mathrm{~g}(66 \%)$; $\mathrm{mp} 187-189{ }^{\circ} \mathrm{C}, \mathrm{R}_{\mathrm{f}}: 0.57$. IR $\left(v_{\max }, \mathrm{KBr}, \mathrm{cm}^{-1}\right)$ : 1600, 1497, 1261. ${ }^{1} \mathrm{H}-\mathrm{NMR}\left(\mathrm{CDCl}_{3}\right): \delta 1.84\left(\mathrm{~m}, 6 \mathrm{H}, \mathrm{CH}_{2}-4, \mathrm{CH}_{2}-6, \mathrm{CH}_{2}-10\right) ; 2.17$ (m, 9H, $\mathrm{CH}_{2}-2$, $\left.\mathrm{CH}-3, \mathrm{CH}-5, \mathrm{CH}-7, \mathrm{CH}_{2}-8, \mathrm{CH}_{2}-9\right) ; 2.46\left(\mathrm{~s}, 3 \mathrm{H}, \mathrm{Ph}-\mathrm{CH}_{3}\right) ; 7.35$ (d, 2H, $J_{2,3}=J_{5,6}=9.0 \mathrm{~Hz}, \mathrm{Ar}-\mathrm{H}-3$, $\mathrm{Ar}-\mathrm{H}-5) ; 8.07\left(\mathrm{~s}, 1 \mathrm{H}, \mathrm{H}^{5}\right.$ thiazole $) ; 8.08(\mathrm{~d}, 2 \mathrm{H}, \mathrm{Ar}-\mathrm{H}-2, \mathrm{Ar}-\mathrm{H}-6) .{ }^{13} \mathrm{C}-\mathrm{NMR}\left(\mathrm{CDCl}_{3}\right): \delta 21.7\left(\mathrm{Ph}-\mathrm{CH}_{3}\right)$; 
28.5, 36.4, 39.9, $43.1\left(\mathrm{C}_{\text {adaman. }}\right) ; 120.7\left(\mathrm{C}^{5}\right.$ thiazole $) ; 121.1,127.1,129.7\left(\mathrm{C}_{\text {arom }}\right) ; 139.5\left(\mathrm{C}^{4}\right.$ thiazole $) ; 142.3$ $\left(\mathrm{C}_{\text {arom. }}^{1}\right) ; 160.6\left(\mathrm{C}_{\text {oxadiazole }}^{2}\right) ; 164.6\left(\mathrm{C}_{\text {oxadiazole }}^{5}\right) ; 183.5\left(\mathrm{C}_{\text {thiazole }}^{2}\right)$. EI-MS (m/z; \%): $377\left(\mathrm{M}^{+\cdot}, 100\right)$, 246 (10), 160 (33), 135 (15), 121 (10), 119 (25), 107 (3), 93 (7), 91 (27), 79 (15), 65 (10). Anal. calcd. for $\mathrm{C}_{22} \mathrm{H}_{23} \mathrm{~N}_{3} \mathrm{SO}$ : C, 69.90; H, 6.14; N, 11.10; S,8.49; Found: C, 69.95; H, 6.29; N, 10.81; S, 8.39 .

2-(2-Adamantyl-1,3-thiazol-4-yl)-5-(3-methylphenyl)-1,3,4-oxadiazole $\quad$ (6b). From 3methylbenzoic acid (0.25 g). Yield: $0.43 \mathrm{~g}(63 \%)$; mp $153-155^{\circ} \mathrm{C} ; \mathrm{R}_{\mathrm{f}}: 0.55$. IR $\left(v_{\max }, \mathrm{KBr}, \mathrm{cm}^{-1}\right)$ : 1590, 1549, 1263. ${ }^{1} \mathrm{H}-\mathrm{NMR}\left(\mathrm{CDCl}_{3}\right): \delta 1.83$ (m, 6H, $\left.\mathrm{CH}_{2}-4, \mathrm{CH}_{2}-6, \mathrm{CH}_{2}-10\right) ; 2.17$ (m, 9H, $\mathrm{CH}_{2}-2$, CH-3, CH-5, CH-7, $\left.\mathrm{CH}_{2}-8, \mathrm{CH}_{2}-9\right) ; 2.47$ (s, 3H, $\left.\mathrm{CH}_{3}\right) ; 7.37$ (d, 1H, J = $\left.7.5 \mathrm{~Hz}, \mathrm{Ar}-\mathrm{H}-4\right) ; 7.43$ (t, $\left.1 \mathrm{H}, J_{5,6}=7.5 \mathrm{~Hz}, \mathrm{Ar}-\mathrm{H}-5\right) ; 7.98(\mathrm{~s}, 1 \mathrm{H}, \mathrm{Ar}-\mathrm{H}-2) ; 8.02$ (s, $1 \mathrm{H}, \mathrm{H}^{5}$ thiazole); 8.08 (d, $\left.1 \mathrm{H}, \mathrm{Ar}-\mathrm{H}-6\right) .{ }^{13} \mathrm{C}-$ NMR $\left(\mathrm{CDCl}_{3}\right): \delta 22.3\left(\mathrm{Ph}-\mathrm{CH}_{3}\right) ; 28.6,36.5,39.9,43.1\left(\mathrm{C}_{\text {adaman. }}\right) ; 120.8\left(\mathrm{C}_{\text {thiazole }}^{5}\right) ; 123.6,124.3$, 127.7, 128.9, $132.6\left(\mathrm{C}_{\text {arom }}\right) ; 139.5\left(\mathrm{C}_{\text {thiazole }}^{4}\right) ; 160.7$ ( $\mathrm{C}^{2}$ oxadiazole $) ; 164.5\left(\mathrm{C}_{\text {oxadiazole }}^{5}\right) ; 183.7\left(\mathrm{C}^{2}\right.$ thiazole $)$. EI-MS (m/z; \%): $377\left(\mathrm{M}^{+*}, 100\right), 246$ (10), 160 (20), 135 (10), 119 (15), 121 (3), 107 (2), 93 (6), 91 (17), 79 (10), 65 (5).

2-(2-Adamantyl-1,3-thiazol-4-yl)-5-(2-methylphenyl)-1,3,4-oxadiazole $\quad$ (6c). From 2methylbenzoic acid (0.25 g). Yield: $0.43 \mathrm{~g}(64 \%)$; $\mathrm{mp} 151-153{ }^{\circ} \mathrm{C} ; \mathrm{R}_{\mathrm{f}}: 0.61$. IR $\left(v_{\max }, \mathrm{KBr}, \mathrm{cm}^{-1}\right)$ : 1601, 1536, 1261. ${ }^{1} \mathrm{H}-\mathrm{NMR}\left(\mathrm{CDCl}_{3}\right): \delta 1.84\left(\mathrm{~m}, 6 \mathrm{H}, \mathrm{CH}_{2}-4, \mathrm{CH}_{2}-6, \mathrm{CH}_{2}-10\right) ; 2.17$ (m, 9H, $\mathrm{CH}_{2}-2$, $\left.\mathrm{CH}-3, \mathrm{CH}-5, \mathrm{CH}-7, \mathrm{CH}_{2}-8, \mathrm{CH}_{2}-9\right)$; 2.78 (s, 3H, Ph- $\mathrm{CH}_{3}$ ); 7.34-7.45 (m, 3H, Ar-H-3, Ar-4, Ar-H5); 8.07 (m, 2H, Ar-H-6, $\mathrm{H}^{5}$ thiazole). ${ }^{13} \mathrm{C}-\mathrm{NMR}\left(\mathrm{CDCl}_{3}\right): \delta 22.1\left(\mathrm{Ph}-\mathrm{CH}_{3}\right) ; 28.5,36.4,39.9,43.1$ $\left(\mathrm{C}_{\text {adaman. }}\right) ; 120.7\left(\mathrm{C}_{\text {thiazole }}^{5}\right) ; 122.9,126.1,129.2,131.2\left(\mathrm{C}_{\text {arom. }}\right) ; 138.6\left(\mathrm{C}_{\text {arom. }}^{2}\right) ; 139.5\left(\mathrm{C}_{\text {thiazole }}^{4}\right)$; $\left.160.4\left(\mathrm{C}_{\text {oxadiazole }}^{2}\right) ; 164.6 \mathrm{C}_{\text {oxadiazole }}^{5}\right) ; 183.6\left(\mathrm{C}^{2}\right.$ thiazole $)$. EI-MS (m/z; \%): $377\left(\mathrm{M}^{+*}, 100\right), 246(13)$, 160 (10), 135 (17), 121 (4), 119 (15), 107 (2), 93 (2), 91 (20), 65 (8).

2-(2-Adamantyl-1,3-thiazol-4-yl)-5-(4-chlorophenyl)-1,3,4-oxadiazole (6d). From 4-chlorobenzoic acid $(0.16 \mathrm{~g})$. Yield: $0.26 \mathrm{~g}(64 \%)$; mp 181-183 ${ }^{\circ} \mathrm{C} ; \mathrm{R}_{\mathrm{f}}: 0.47$. IR $\left(v_{\max }, \mathrm{KBr}, \mathrm{cm}^{-1}\right): 1596$, 1543, 1262, 1019. ${ }^{1} \mathrm{H}-\mathrm{NMR}\left(\mathrm{CDCl}_{3}\right): \delta 1.83\left(\mathrm{~m}, 6 \mathrm{H}, \mathrm{CH}_{2}-4, \mathrm{CH}_{2}-6, \mathrm{CH}_{2}-10\right) ; 2.16\left(\mathrm{~m}, 9 \mathrm{H}, \mathrm{CH}_{2}-2\right.$, CH-3, CH-5, CH-7, $\left.\mathrm{CH}_{2}-8, \mathrm{CH}_{2}-9\right) ; 7.52$ (d, 2H, $\left.J_{2,3}=J_{5,6}=8.7 \mathrm{~Hz}, \mathrm{Ar}-\mathrm{H}-2, \mathrm{Ar}-\mathrm{H}-6\right) ; 8.09$ (s, 1H, $\mathrm{H}^{5}$ thiazol $) ; 8.13(\mathrm{~d}, 2 \mathrm{H}, \mathrm{Ar}-\mathrm{H}-3, \mathrm{Ar}-\mathrm{H}-5) .{ }^{13} \mathrm{C}-\mathrm{NMR}\left(\mathrm{CDCl}_{3}\right): \delta 28.5,36.4,39.9,43.1\left(\mathrm{C}_{\text {adaman. }}\right) ; 121.1$ $\left(\mathrm{C}^{5}\right.$ thiazole $) ; 122.3,128.4,129.4\left(\mathrm{C}_{\text {arom }}\right) ; 134.9\left(\mathrm{C}_{\text {arom. }}^{4}\right) ; 139.2\left(\mathrm{C}_{\text {thiazole }}^{4}\right) ; 160.9\left(\mathrm{C}_{\text {oxadiazole }}^{2}\right) ; 163.6$ $\left(\mathrm{C}^{5}\right.$ oxadiazole $) ; 183.8\left(\mathrm{C}_{\text {thiazole }}^{2}\right)$. EI-MS (m/z; \%): $399(\mathrm{M}+2,33), 397\left(\mathrm{M}^{+*}, 100\right), 246(12), 217(4)$, 182 (5), 180 (14), 141 (5), 139 (16), 135 (15), 121 (1), 113 (4), 111(12), 107 (2), 93 (12), 79 (15).

2-(2-Adamantyl-1,3-thiazol-4-yl)-5-(3-chlorophenyl)-1,3,4-oxadiazole $\quad$ (6e). From 3chlorobenzoic acid $(0.16 \mathrm{~g})$. Yield: $0.24 \mathrm{~g}(61 \%)$; mp $165-168{ }^{\circ} \mathrm{C} ; \mathrm{R}_{\mathrm{f}}: 0.52$. IR $\left(v_{\max }, \mathrm{KBr}, \mathrm{cm}^{-1}\right)$ : 1576, 1547, 1262, 1086. ${ }^{1} \mathrm{H}-\mathrm{NMR}\left(\mathrm{CDCl}_{3}\right): \delta 1.83\left(\mathrm{~m}, 6 \mathrm{H}, \mathrm{CH}_{2}-4, \mathrm{CH}_{2}-6, \mathrm{CH}_{2}-10\right) ; 2.16(\mathrm{~m}, 9 \mathrm{H}$, $\left.\mathrm{CH}_{2}-2, \mathrm{CH}-3, \mathrm{CH}-5, \mathrm{CH}-7, \mathrm{CH}_{2}-8, \mathrm{CH}_{2}-9\right)$; 7.31-7.55 (m, 2H, Ar-H-4, Ar-H-5), 8.00-8.21 (m, 3H, Ar-H-2, Ar-H-6, $\left.\mathrm{H}_{\text {thiazole }}^{5}\right){ }^{13} \mathrm{C}-\mathrm{NMR}\left(\mathrm{CDCl}_{3}\right): \delta$ 28.6, 36.2, 39.9, $43.0\left(\mathrm{C}_{\text {adaman. }}\right) ; 121.3\left(\mathrm{C}^{5}\right.$ thiazole $)$; 125.3, 125.4, 127.1, 130.4, 131.8, 135 ( $\left.\mathrm{C}_{\text {arom }}\right)$; $\left(\mathrm{C}^{3}\right.$ arom. $)$; $139.1\left(\mathrm{C}_{\text {thiazole }}^{4}\right) ; 160.5\left(\mathrm{C}_{\text {oxadiazole }}^{2}\right) ; 163.2$, $\left(\mathrm{C}^{5}\right.$ oxadiazole $) ; 183.8\left(\mathrm{C}^{2}\right.$ thiazole $)$. EI-MS (m/z; \%): $399(\mathrm{M}+2,33), 397\left(\mathrm{M}^{+*}, 100\right), 246(10), 217(2)$, 182 (2), 180 (7), 141 (5), 139 (16), 135 (15), 121 (1), 113 (4), 111(13), 107 (2), 93 (14), 79 (18).

2-(2-Adamantyl-1,3-thiazol-4-yl)-5-(2-chlorophenyl)-1,3,4-oxadiazole $\quad(6 f) . \quad$ From 2chlorobenzoic acid $(0.16 \mathrm{~g})$. Yield: $0.24 \mathrm{~g}(62 \%)$; mp $148-150{ }^{\circ} \mathrm{C} ; \mathrm{R}_{\mathrm{f}}: 0.50$. IR $\left(v_{\max }, \mathrm{KBr}, \mathrm{cm}^{-1}\right)$ : 
1596, 1532, 1262, 1088. ${ }^{1} \mathrm{H}-\mathrm{NMR}\left(\mathrm{CDCl}_{3}\right): \delta 1.82\left(\mathrm{~m}, 6 \mathrm{H}, \mathrm{CH}_{2}-4, \mathrm{CH}_{2}-6, \mathrm{CH}_{2}-10\right) ; 2.16(\mathrm{~m}, 9 \mathrm{H}$, $\mathrm{CH}_{2}-2$, CH-3, CH-5, CH-7, $\left.\mathrm{CH}_{2}-8, \mathrm{CH}_{2}-9\right) ; 7.44$ (dt, $1 \mathrm{H}, J_{4,5}=J_{3,4}=7.7 \mathrm{~Hz}, J_{4,6}=1.5 \mathrm{~Hz}$, Ar-H-4); $7.50(\mathrm{~m}, 1 \mathrm{H}, \mathrm{Ar}-\mathrm{H}-5) ; 7.58$ (dd, $\left.1 \mathrm{H}, J_{3,4}=7.7 \mathrm{~Hz}, J_{3,5}=1.5 \mathrm{~Hz}, \mathrm{Ar}-\mathrm{H}-3\right), 8.09$ (dd, $1 \mathrm{H}, J_{5,6}=7.5$ $\left.\mathrm{Hz}, J_{4,6}=1.8 \mathrm{~Hz}, \mathrm{H}-6\right), 8.09$ (s, $1 \mathrm{H}, \mathrm{H}^{5}$ thiazole $) .{ }^{13} \mathrm{C}-\mathrm{NMR}\left(\mathrm{CDCl}_{3}\right): \delta 28.5,36.4,39.9,43.1\left(\mathrm{C}_{\text {adaman. }}\right)$; $121.3\left(\mathrm{C}_{\text {thiazole }}^{5}\right) ; 123.2,127.1,131.1,131.5,132.4\left(\mathrm{C}_{\text {arom }}\right) ; 133.3\left(\mathrm{C}_{\text {arom. }}^{1}\right) ; 139.3\left(\mathrm{C}_{\text {thiazole }}^{4}\right) ; 161.2$ $\left.\left(\mathrm{C}_{\text {oxadiazole }}^{2}\right) ; 162.7 \mathrm{C}_{\text {oxadiazole }}^{5}\right) ; 183.8\left(\mathrm{C}_{\text {thiazole }}^{2}\right)$. EI-MS (m/z; \%): $399(\mathrm{M}+2,33), 397\left(\mathrm{M}^{+}, 100\right)$, 246 (14), 182 (3), 180 (10), 141 (5), 139 (16), 135 (15), 121 (1), 113 (3), 111(10), 107 (2), 93 (15), 79 (18).

2-(2-Adamantyl-1,3-thiazol-4-yl)-5-(4-bromophenyl)-1,3,4-oxadiazole $\quad$ (6g). $\quad$ From 4bromobenzoic acid $(0.20 \mathrm{~g})$. Yield: $0.27 \mathrm{~g}(61 \%) ; \mathrm{mp} 188-190{ }^{\circ} \mathrm{C}$; $\mathrm{R}_{\mathrm{f}}: 0.53$. IR $\left(v_{\max }, \mathrm{KBr}, \mathrm{cm}^{-1}\right)$ : 1593, 1474, 1102, 1075; ${ }^{1} \mathrm{H}-\mathrm{NMR}\left(\mathrm{CDCl}_{3}\right): \delta 1.83\left(\mathrm{~m}, 6 \mathrm{H}, \mathrm{CH}_{2}-4, \mathrm{CH}_{2}-6, \mathrm{CH}_{2}-10\right) ; 2.15(\mathrm{~m}, 9 \mathrm{H}$, $\mathrm{CH}_{2}-2, \mathrm{CH}-3, \mathrm{CH}-5, \mathrm{CH}-7, \mathrm{CH}_{2}-8, \mathrm{CH}_{2}-9$ ); 7.67 (d, 2H, $J_{2,3}=J_{5,6}=8.6 \mathrm{~Hz}, \mathrm{Ar}-\mathrm{H}-2$, Ar-H-6); 8.04 (s, $\left.1 \mathrm{H}, \mathrm{H}_{\text {thiazol }}^{5}\right) ; 8.04$ (d, 2H, Ar-H-3, Ar-H-5). ${ }^{13} \mathrm{C}-\mathrm{NMR}\left(\mathrm{CDCl}_{3}\right): \delta 28.5,36.3,39.9,43.0$, $\left(\mathrm{C}_{\text {adaman }}\right) ; 121.1\left(\mathrm{C}_{\text {thiazole }}^{5}\right) ; 122.7,126.4,128.5\left(\mathrm{C}_{\text {arom }}\right) ; 132.3\left(\mathrm{C}_{\text {arom. }}^{4}\right) ; 139.2\left(\mathrm{C}_{\text {thiazole }}^{4}\right) ; 160.9$ $\left(\mathrm{C}_{\text {oxadiazole }}^{2}\right) ; 163.6\left(\mathrm{C}_{\text {oxadiazole }}^{5}\right) ; 183.7\left(\mathrm{C}_{\text {thiazole }}^{2}\right.$. EI-MS (m/z; \%): $443(\mathrm{M}+2,100), 441\left(\mathrm{M}^{+}, 100\right)$, 362 (5), 246 (12), 217 (2), 185 (14), 183 (15), 157 (10), 155 (9), 135 (20), 121 (5), 107 (7), 93 (18), 79 (25).

2-(2-Adamantyl-1,3-thiazol-4-yl)-5-(3-bromophenyl)-1,3,4-oxadiazole $\quad$ (6h). From 3bromobenzoic acid $(0.20 \mathrm{~g})$. Yield: $0.28 \mathrm{~g}(63 \%) ; \mathrm{mp} 171-173{ }^{\circ} \mathrm{C}$; R: $\mathrm{R}_{\mathrm{f}} 0.57$; IR $\left(v_{\max }, \mathrm{KBr}, \mathrm{cm}^{-1}\right)$ : 1590, 1545, 1260, 1084; ${ }^{1} \mathrm{H}-\mathrm{NMR}\left(\mathrm{CDCl}_{3}\right): \delta 1.84\left(\mathrm{~m}, 6 \mathrm{H}, \mathrm{CH}_{2}-4, \mathrm{CH}_{2}-6, \mathrm{CH}_{2}-10\right) ; 2.16(\mathrm{~m}, 9 \mathrm{H}$, $\left.\mathrm{CH}_{2}-2, \mathrm{CH}-3, \mathrm{CH}-5, \mathrm{CH}-7, \mathrm{CH}_{2}-8, \mathrm{CH}_{2}-9\right) ; 7.42$ (t, $\left.1 \mathrm{H}, J_{5,6}=7.8 \mathrm{~Hz}, \mathrm{Ar}-\mathrm{H}-5\right) ; 7.69$ (m, 2H, Ar-H2, Ar-H-4); 8.10 (m, $1 \mathrm{H}, \mathrm{Ar}-\mathrm{H}-6) ; 8.12\left(\mathrm{~s}, 1 \mathrm{H}, \mathrm{H}_{\text {thiazole }}^{5}\right){ }^{13} \mathrm{C}-\mathrm{NMR}\left(\mathrm{CDCl}_{3}\right): \delta 28.5,36.4,39.9,43.0$ $\left(\mathrm{C}_{\text {adaman. }}\right) ; 121.2\left(\mathrm{C}_{\text {thiazole }}^{5}\right) ; 123.0,125.7,129.9,130.5,134.6\left(\mathrm{C}_{\text {arom. }}\right) ; 139.1\left(\mathrm{C}_{\text {thiazole }}^{4}\right) ; 161.0$ $\left(\mathrm{C}_{\text {oxadiazole }}^{2}\right) ; 163.0$ ( $\left.\mathrm{C}_{\text {oxadiazole }}^{5}\right) ; 183.8\left(\mathrm{C}_{\text {thiazole }}^{2}\right.$. EI-MS (m/z; \%): $443(\mathrm{M}+2,100), 441\left(\mathrm{M}^{+}, 100\right)$, 362 (5), 246 (10), 217 (10), 185 (10), 183 (10), 157 (12), 155 (12), 135 (18), 121 (3), 107 (10), 93 (19), 79 (25).

2-(2-Adamantyl-1,3-thiazol-4-yl)-5-(2-bromophenyl)-1,3,4-oxadiazole (6i). From 2bromobenzoic acid $(0.20 \mathrm{~g})$. Yield: $0.28 \mathrm{~g}(64 \%)$; mp 186-188 ${ }^{\circ} \mathrm{C}$; Rf: 0.51 . IR $\left(v_{\max }, \mathrm{KBr}, \mathrm{cm}^{-1}\right)$ : 1592, 1489, 1262, 1013. ${ }^{1} \mathrm{H}-\mathrm{NMR}\left(\mathrm{CDCl}_{3}\right): \delta 1.83\left(\mathrm{~m}, 6 \mathrm{H}, \mathrm{CH}_{2}-4, \mathrm{CH}_{2}-6, \mathrm{CH}_{2}-10\right) ; 2.16(\mathrm{~m}, 9 \mathrm{H}$, $\mathrm{CH}_{2}-2$, CH-3, CH-5, CH-7, $\left.\mathrm{CH}_{2}-8, \mathrm{CH}_{2}-9\right) ; 7.41$ (m, 1H, Ar-H-4); 7.49 (m, 1H, Ar-H-5); 7.78 (m, $2 \mathrm{H}, \mathrm{Ar}-\mathrm{H}-3$, Ar-H-6); 8.09 (s, $\left.1 \mathrm{H}, \mathrm{H}_{\text {thiazole }}^{5}\right) .{ }^{13} \mathrm{C}-\mathrm{NMR}\left(\mathrm{CDCl}_{3}\right): \delta 28.5,36.4,39.9,43.0\left(\mathrm{C}_{\text {adaman. }}\right)$; $121.2\left(\mathrm{C}_{\text {thiazole }}^{5}\right) ; 121.8\left(\mathrm{C}_{\text {arom }}^{2}\right) ; 125.3,127.5,131.8,132.5\left(\mathrm{C}_{\text {arom. }}\right) ; 134.4\left(\mathrm{C}_{\text {arom }}^{1}\right) ; 139.2\left(\mathrm{C}_{\text {thiazole }}^{4}\right)$; 161.2 ( $\left.\mathrm{C}_{\text {oxadiazole }}^{2}\right) ; 163.6\left(\mathrm{C}_{\text {oxadiazole }}^{5}\right) ; 183.8\left(\mathrm{C}_{\text {thiazole }}^{2}\right)$. EI-MS (m/z; \%): $443(\mathrm{M}+2,98), 441\left(\mathrm{M}^{+*}\right.$, 100), 362 (10), 246 (20), 217 (10), 185 (15), 183 (15), 157 (10), 155 (10), 135 (25), 121 (3), 107 (10), 93 (19), 79 (22).

2-(2-Adamantyl-1,3-thiazol-4-yl)-5-(4-flourophenyl)-1,3,4-oxadiazole $\quad$ (6j). $\quad$ From 4fluorobenzoic acid $(0.14 \mathrm{~g})$. Yield: $0.24 \mathrm{~g}(63 \%)$; mp 213-215 ${ }^{\circ} \mathrm{C} ; \mathrm{R}_{\mathrm{f}}: 0.47$. IR $\left(v_{\max }, \mathrm{KBr}, \mathrm{cm}^{-1}\right)$ : 1606, 1497, 1262, 1222. ${ }^{1} \mathrm{H}-\mathrm{NMR}\left(\mathrm{CDCl}_{3}\right): \delta 1.83\left(\mathrm{~m}, 6 \mathrm{H}, \mathrm{CH}_{2}-4, \mathrm{CH}_{2}-6, \mathrm{CH}_{2}-10\right) ; 2.16(\mathrm{~m}, 9 \mathrm{H}$, $\left.\mathrm{CH}_{2}-2, \mathrm{CH}-3, \mathrm{CH}-5, \mathrm{CH}-7, \mathrm{CH}_{2}-8, \mathrm{CH}_{2}-9\right) ; 7.23$ (t, $2 \mathrm{H}, J=8.4 \mathrm{~Hz}$, Ar-H-3, Ar-H-5); 8.08 (s, 1H, $\left.\mathrm{H}_{\text {thiazol }}^{5}\right) ; 8.20\left(\mathrm{dd}, 2 \mathrm{H}, J_{2^{\prime}, 3^{\prime}}=9.0 \mathrm{~Hz}, J_{2^{\prime}, 6^{\prime}}=5.4 \mathrm{~Hz}, \mathrm{Ar}-\mathrm{H}-2, \mathrm{Ar}-\mathrm{H}-6\right) .{ }^{13} \mathrm{C}-\mathrm{NMR}\left(\mathrm{CDCl}_{3}\right): \delta 28.5$, 
36.4, 39.9, $43.0\left(\mathrm{C}_{\text {adaman. }}\right) ; 116.3\left(\mathrm{~d}, J_{C, F}=22.5 \mathrm{~Hz}, \mathrm{C}^{3,5}\right.$ arom $) ; 120.1\left(\mathrm{C}^{5}{ }_{\text {thiazol. }}\right) ; 129.8\left(\mathrm{~d}, J_{C, F}=9.0\right.$ $\mathrm{Hz}, \mathrm{C}^{2,6}$ arom. $) ; 139.3\left(\mathrm{C}_{\text {thiazole }}^{4}\right) ; 161.0\left(\mathrm{C}_{\text {oxadiazole }}^{2}\right) ; 163.1\left(\mathrm{C}_{\text {oxadiazole }}^{5}\right) ; 163.7\left(\mathrm{~d}, J_{C, F}=252.2 \mathrm{~Hz}\right.$, $\left.\mathrm{C}_{\text {arom. }}^{4}\right) ; 183.8$ ( $\mathrm{C}_{\text {thiazole }}^{2}$. EI-MS (m/z; \%): $381\left(\mathrm{M}^{++}, 100\right), 246$ (11), 217 (2), 164 (15), 135 (10), 121 (4), 107 (4), 95 (7), 93 (6), 79 (8).

2-(2-Adamantyl-1,3-thiazol-4-yl)-5-(3-flourophenyl)-1,3,4-oxadiazole $\quad$ (6k). From 3fluorobenzoic acid $(0.14 \mathrm{~g})$. Yield: $0.25 \mathrm{~g}(66 \%) ; \mathrm{mp} 158-160^{\circ} \mathrm{C}$; $\mathrm{R}_{\mathrm{f}}: 0.52$. IR $\left(v_{\max }, \mathrm{KBr}, \mathrm{cm}^{-1}\right)$ : 1587, 1551, 1259, 1225; ${ }^{1} \mathrm{H}-\mathrm{NMR}\left(\mathrm{CDCl}_{3}\right): \delta 1.82\left(\mathrm{~m}, 6 \mathrm{H}, \mathrm{CH}_{2}-4, \mathrm{CH}_{2}-6, \mathrm{CH}_{2}-10\right) ; 2.16(\mathrm{~m}, 9 \mathrm{H}$, $\left.\mathrm{CH}_{2}-2, \mathrm{CH}-3, \mathrm{CH}-5, \mathrm{CH}-7, \mathrm{CH}_{2}-8, \mathrm{CH}_{2}-9\right) ; 7.26\left(\mathrm{dt}, 1 \mathrm{H}, J_{4,5}=8.1 \mathrm{~Hz}, J_{4,6}=2.4 \mathrm{~Hz}, J_{4, F}=8.3 \mathrm{~Hz}\right.$, Ar-H-4), 7.52 (m, 2H, Ar-H-2, Ar-H-5), 7.88 (s, 1H, H thiazol); 7.99 (m, 1H, Ar-H-6). ${ }^{13} \mathrm{C}-\mathrm{NMR}$ $\left(\mathrm{CDCl}_{3}\right): \delta 28.5,36.4,39.9,43.0\left(\mathrm{C}_{\text {adaman. }}\right) ; 113.6\left(\mathrm{C}_{\text {thiazole }}^{5}\right) ; 114.1\left(\mathrm{~d}, J_{C 2, F}=24.0 \mathrm{~Hz}, \mathrm{C}_{\text {arom. }}^{2}\right)$; $115.3\left(\mathrm{~d}, J_{C 4, F}=21.0 \mathrm{~Hz}, \mathrm{C}^{4}{ }_{\text {arom. }}\right) ; 122.9\left(\mathrm{~d}, J_{C 6, F}=3.0 \mathrm{~Hz}, \mathrm{C}^{6}{ }_{\text {arom. }}\right) ; 125.6\left(\mathrm{~d}, J_{C 5, F}=8.2 \mathrm{~Hz}, \mathrm{C}_{\text {arom. }}^{5}\right.$ ); $130.8\left(\mathrm{~d}, J_{C l, F}=7.1 \mathrm{~Hz}, \mathrm{C}_{\text {arom.. }}^{1}\right) ; 161.1\left(\mathrm{C}^{2}{ }_{\text {oxadiazole }}\right) ; 162.8\left(\mathrm{~d}, J_{C 2, F}=246.0 \mathrm{~Hz}, \mathrm{C}_{\text {arom }}^{3}\right) ; 163.3$ $\left(\mathrm{C}_{\text {oxadiazole }}^{5}\right) ; 183.8\left(\mathrm{C}_{\text {thiazole }}^{2}\right.$ ). EI-MS (m/z; \%): $381\left(\mathrm{M}^{+*}, 100\right), 246(11), 217$ (2), 164 (15), $135(10)$, 121 (4), 107 (4), 95 (7), 93 (6), 79 (8).

2-(2-Adamantyl-1,3-thiazol-4-yl)-5-(2-flourophenyl)-1,3,4-oxadiazole (6l). From 2-flurobenzoic acid $(0.14 \mathrm{~g})$. Yield: $0.25 \mathrm{~g}(65 \%)$; $\mathrm{mp} 175-177^{\circ} \mathrm{C}$; $\mathrm{R}_{\mathrm{f}}$ : 0.53. IR $\left(v_{\max }, \mathrm{KBr}, \mathrm{cm}^{-1}\right): 1596,1467,1256$, 1228. ${ }^{1} \mathrm{H}-\mathrm{NMR}\left(\mathrm{CDCl}_{3}\right): \delta 1.82\left(\mathrm{~m}, 6 \mathrm{H}, \mathrm{CH}_{2}-4, \mathrm{CH}_{2}-6, \mathrm{CH}_{2}-10\right) ; 2.15$ (m, 9H, $\mathrm{CH}_{2}-2, \mathrm{CH}-3, \mathrm{CH}-5$, CH-7, $\left.\mathrm{CH}_{2}-8, \mathrm{CH}_{2}-9\right)$; 7.24-7.34 (m, 2H, Ar-H-3, Ar-H-5); 7.59 (m, 1H, Ar-H-4), 8.09 (s, 1H, $\left.\mathrm{H}_{\text {thiazole }}^{5}\right) ; 8.17\left(\mathrm{t}, 1 \mathrm{H}, J_{H 6, F}=8.4 \mathrm{~Hz}, \mathrm{Ar}-\mathrm{H}-6\right) .{ }^{13} \mathrm{C}-\mathrm{NMR}\left(\mathrm{CDCl}_{3}\right): \delta 28.5,36.4,39.9,43.0$, $\left(\mathrm{C}_{\text {adaman. }}\right) ; 112.3\left(\mathrm{C}_{\text {thiazole }}^{5}\right) ; 116.9\left(\mathrm{~d}, J_{C 3, F}=21.0 \mathrm{~Hz}, \mathrm{C}_{\text {arom. }}^{3}\right) ; 124.6\left(\mathrm{~d}, J_{C l, F}=21.2 \mathrm{~Hz}, \mathrm{C}_{\text {arom }}^{1}\right)$; $130.0\left(\mathrm{~d}, J_{C \zeta, F}=3.7 .0 \mathrm{~Hz}, \mathrm{C}_{\text {arom. }}^{5}\right) ; 133.5\left(\mathrm{~d}, J_{C 6, F}=9.0 \mathrm{~Hz}, \mathrm{C}_{\text {arom. }}^{6}\right) ; 139.2\left(\mathrm{~d}, J_{C 4, F}=9.2 \mathrm{~Hz}\right.$, $\left.\mathrm{C}_{\text {arom. }}^{4}\right) ; 160.0\left(\mathrm{~d}, J_{C 2, F}=257.2 \mathrm{~Hz}, \mathrm{C}_{\text {arom. }}^{2}\right) ; 161.0\left(\mathrm{C}_{\text {oxadiazole }}^{2}\right) ; 161.1\left(\mathrm{C}_{\text {oxadiazole }}^{5}\right) ; 183.7\left(\mathrm{C}_{\text {thiazole }}^{2}\right)$. EI-MS (m/z; \%): 381 (M+*, 100), 246 (11), 217 (2), 164 (15), 135 (10), 121 (4), 107 (4), 95 (7), 93 (6), $79(8)$.

\section{Cytotoxicity assays}

Cell cultures were seeded at $1 \times 10^{5}$ cells $/ \mathrm{mL}$ in 96 multiwell plates in specific media supplemented (5\%) atmosphere supplemented with 10\% FCS and antibiotics then incubated atand antibiotics and incubated at $37{ }^{\circ} \mathrm{C}$ in a humidified $\mathrm{CO}_{2}$ in the absence or presence of serial dilutions of test compounds. Cell viability was determined after $96 \mathrm{hrs}$ at $37^{\circ} \mathrm{C}$ by the 3-(4,5-dimethylthiazol-2-yl)2,5-diphenyl-tetrazolium bromide (MTT) method. ${ }^{25}$

Compounds were dissolved in DMSO at $100 \mathrm{mM}$ and then diluted into culture medium.

\section{References}

1. Davies, W. L.; Grunnert, R. R.; Haff, R. F.; McGahen, J. W.; Neumeyer, E. M.; Paulshock, M.; Watts, J. C.; Wood, T. R.; Hermann, E. C.; Hoffmann, C. E. Science 1964, 144, 862.

2. Togo, Y.; Hornick, R. B.; Dawkins. A. T. J. Am. Med. Assoc. 1968, 203, 1089.

3. Wendel, H. A.; Snyder, M. T.; Pell. S. Clin. Pharmacol. Therap. 1966, 7, 38. 
4. Moody, T. W.; Jensen, R. T. J. Pharmacol. Exp. Ther. 2001, 299, 1154.

5. Ru, Y.; Marquis, R. W.; Veber, D. F. U.S. Patent 6369 077, 2002; Chem. Abstr. 2002, 130, 4263c.

6. Van Derpoorten, K.; Balzarini, J.; De Clercq, E.; Poupaert. J. H.; Biomed. Pharmacother. 1997, $51,464$.

7. Balzarini, J.; Orzeszko, B.; Maurin, J. K.; Orzeszko. A. Eur. J. Med. Chem. 2007, 42, 993.

8. Pedersen, O. E.; Pedersen, E. B. Antiviral Chem. Chemother. 1999, 10, 285.

9. Burstein, M. E.; Serbin, A. V.; Khakhulina, T. V.; Alymova, I. V.; Stotskaya, L. L.; Bogdan, O. P.; Manukchina, E. E.; Jdanov, V. V.; Sharova, N. K. Antiviral Res. 1999, 41, 135.

10. Maynard, M.; Pradat, P.; Bailly, F.; Rozier, F.; Nemoz, C.; Ahmed, S. N.; Adeliene, P.; Trépo, C. J. Hepatol. 2006, 44, 484.

11. Antoniadou-Vyza, E.; Avramidis, N.; Kourounakis, A.; Hadjipetrou. L. Arch. Pharm. 1999, $331,72$.

12. Baxter, A.; Bent, J.; Bowers, K.; Braddock, M.; Brough, S.; Fagura, M.; Lawson, M.; McInally, T.; Mortimore, M.; Robertson, M.; Weaver, R.; Webborn, P. Bioorg. Med. Chem. Lett. 2003, 13, 4047.

13. Georgiev, V. S.; Mullen, G. B.; Acker, C. G. Heterocycles 1986, 24, 751.

14. El-Emam, A. A.; Ibrahim, T. M. Arzneim.-Forsch. Drug Res. 1991, 41, 1260.

15. Al-Deeb, 1. O. A.; Al-Omar, M. A.; El-Brollosy, N. R.; Habib, E. E.; Ibrahim, T. M.; El-Emam, A. A. Arzneim.-Forsch. Drug Res. 2006, 56, 40.

16. Antoniadou-Vyza, E.; Tsitsa, P.; Hytiroglou, E.; Tsantili-Kakoulidou, A. Eur. J. Med. Chem. 1996, 31, 105.

17. Kadi, A. A.; El-Brollosy, N. R.; Al-Deeb, O. A.; Habib, E. E.; Ibrahim, T. M.; El-Emam, A. A. Eur. J. Med. Chem. 2007, 42, 235.

18. Rostom, S. A. F.; Shalaby, M. A.; El-Demellawy, M. A. Eur. J. Med.Chem. 2003, 38, 959.

19. Narayanan V. L. J. Med. Chem. 1972, 15, 682.

20. Balkovec, J. M.; Thieringer, R.; Mundt, S. S.; Hermanowski-Vosatka, A.; Graham, D. W.; Donald, W.; Patel, G. F.; Susan, S. D.; Waddell, S. T.; Sherman, T.; Olson, S. H.; Maletic, M. PCT Int. Appl. WO 20030814, 2003 [Chem. Abstr. 139, 180065].

21. Akhtar, T.; Hameed, S.; Khan, K. M.; Choudhary, M. I. Med. Chem. 2008, 4, 539.

22. Akhtar, T.; Hameed, S.; Al-Masoudi, N. A.; Loddo, R.; La Colla, P. Acta. Pharm. 2008, 58, 135.

23. Akhtar, T.; Hameed, S.; Al-Masoudi, N. A.; Khan, K. M. Heteroatom. Chem. 2007, 18, 316.

24. Willker, W.; Leibfritz, D.; Kerssebaum, R.; Bermel, W. Mag. Reson. Chem. 1993, 31, 287.

25. Alley, C.; Scudiero, D. A.; Monks, A.; Hursey, M. L.; Czerwinski, M. J.; Fine, D. L.; Abbott, B. J.; Mayo, J. G.; Schoemaker, R. H.; Boyd, M. R. F. Cancer Res. 1988, 48, 589. 